\title{
Fuzzy-GMC Control of Gas-Phase Propylene Copolymerization in Fluidized Bed Reactor
}

\author{
Nazratul Fareha Salahuddin ${ }^{1}$, Ahmad Shamiri ${ }^{2}$, Mohd Azlan Hussain ${ }^{1, *}$ and Navid Mostoufi ${ }^{3}$ \\ ${ }^{1}$ Chemical Engineering Department, Faculty of Engineering, University Malaya, 50603 Kuala Lumpur, Malaysia. \\ ${ }^{2}$ Chemical \& Petroleum Engineering Department, Faculty of Engineering, Technology and Built Environment, UCSI University, \\ 56000 Kuala Lumpur, Malaysia. \\ ${ }^{3}$ Multiphase Systems Research Lab. School of Chemical Engineering, College of Engineering, University of Tehran, P.O. Box \\ 11155/4563, Tehran, Iran.
}

\begin{abstract}
A modified two-phase model for gas phase propylene and ethylene copolymerization was chosen to represent the process in a fluidized bed reactor. This model considered the entrainment of solid particles in the reactor, as a modification to the original two-phase model assumptions. The non-linearity of this process makes it difficult to control just by using conventional controller such as PID. A hybrid control strategy (a simple designed fuzzy logic controller (FLC) integrated with generic model control $(\mathrm{GMC})$ ) is designed to control the temperature of the reactor. This advanced control system was compared with the GMC and conventional PID controller. The simulation results showed that the hybrid controller (FuzzyGMC) performed better than both GMC and PID in terms of both servo and regulatory control.
\end{abstract}

\section{Introduction}

Polymerization is a process of linking monomer units by chemical reaction to form long chains called polymer. The process is very difficult to measure let alone to control due to its complex reaction kinetics and physical mechanism [1]. There are many phases involve in the reaction hence lead to various heat and mass transfer mechanism in every phase and also the unusual flow characteristics that need to be considered in order to obtain a good model. All these complexity results to high non-linearity of the process dynamics and this are where the process control challenges come in. The difficulty also found in the on-line monitoring of the product (polymer) quality because of the main quality variables such as composition and molecular weight distribution cannot be measured on-line. If it is at all possible, there are still other problems associated with the measurement such as sampling problems, large dead times and/or high noise levels [2].

This present work will focus on the copolymerization of propylene and ethylene in fluidized bed reactor (FBR) with two sites Ziegler-Natta catalyst and triethyl aluminium as co-catalyst. The utilization of FBR in producing polypropylene (PP) is widely preferable in the polymerization industry. The strong interaction between the reactor variables gave additional complexity to the fundamental control problem. Advanced control strategies are needed to cope these problems of which conventional controller are incapable to handle [3].
Although the studies on the modeling and control of polymerization process in fluidized bed reactor are available in the literature [2, 4-6], but minimal work has been done on the polymerization of propylene, specifically copolymerization of propylene and ethylene, for both modeling and control.

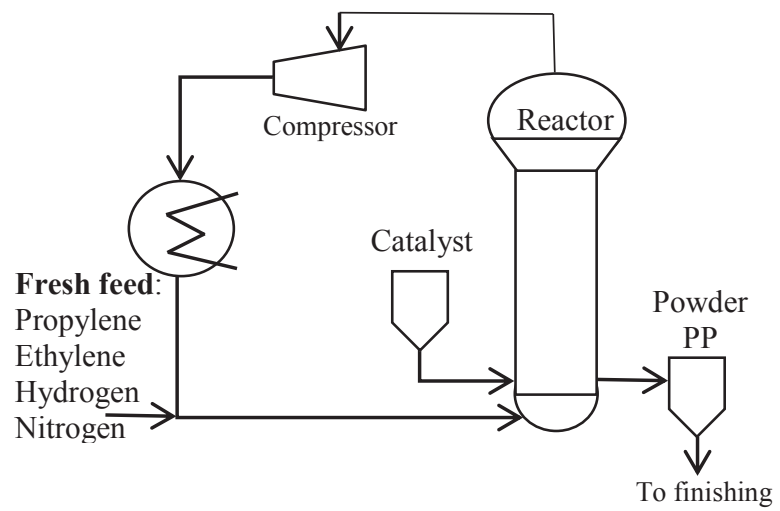

Fig 1. Simplified schematic diagram of fluidized bed propylene and ethylene copolymerization reactor

\footnotetext{
Corresponding author: mohd_azlan@um.edu.my
} 
Figure 1Error! Reference source not found. shows the simplified schematic of fluidized bed propylene and ethylene copolymerization reactor. The feed gas stream (propylene, ethylene, hydrogen and nitrogen) will help to fluidize the growing polymer particles inside the reactor through the distributor along with the injected catalyst. The solid free gas exits from the top of the reactor, compressed and chilled before combined with fresh feed and fed back to the gas distributor. This recirculation of gas will help to remove the heat of polymerization process. The polymer product is withdrawn continuously from the reactor. The heat removal is crucial since the process is highly exothermic. Hence, it is important to stabilize the reactor temperature above the dew point in order to achieve not only optimal production rate but also to prevent agglomeration of polymer and reactor thermal runaway.

A good model will still need a good controller in order to provide a better environment for the system, especially when controlling the reactor temperature to avoid any explosion in extreme cases. Since polymerization process is very well known for its nonlinearity, the searching for the most suitable controller are still going on from time to time. There are many factors influenced the dynamic and steady-state of the polymerization reactor including superficial gas velocity, monomer concentration, monomer feed rate, feed gas temperature and catalyst feed rate among others.

For a polyethylene fluidized bed reactor, Dadebo et al. (1997) [7] used a non-linear error trajectory control (ETC) with SISO system to control the temperature of the reactor. The controller exhibited greater performance and was able to maintain the stability over a wide range of operating conditions compared to optimally tuned PID controller. E. Ali et al. (2003) [8] presented two control schemes in the paper to study the multivariable control of polyethylene in fluidized bed reactor where both nonlinear and linear MPC are used and compared. It was found that non-linear MPC was better than the latter in the sense providing a good performance even in the presence of moderate modeling error. Ghasem (2006) [9] had done a study on fuzzy-logic control for temperature regulatory in polyethylene fluidized bed reactor. Both Takagi-Sugeno (TS) and Mamdani FLC, along with PI controller and hybrid of TS-PI and Mamdani-PI are compared. The results showed that hybrid controllers performed better than advanced controller (FLC) and even better than conventional PI controller. In order to control a liquid pool polypropylene hollow-shaft reactor, M. A.-H. Ali et al. (2007) [2] suggested a modified GMC. The modified controller gave better performance for set point and disturbances changes when compared to GMC and PI-Smith predictor controller. Ahmmed S. Ibrehem et al. (2008) [4] studied the performance of neural-network based predictive controller for temperature control of ethylene copolymerization fluidized bed reactor. By using modified three-phase model, the neural-network based MPC displayed greater performance in handling servo and regulatory control than PID. To control molecular weight distribution (MWD), E. M. Ali and Ali (2010) [10] designed nonlinear MPC to manipulate hydrogen flow and catalysts feed rate. The non-linear predictive control managed to give a good performance even when there is modeling errors. Adaptive Predictive Model-based Control (APMBC) is proposed by Ho et al. (2012) [3] to control both emulsion temperature and production rate of a polypropylene fluidized bed reactor. The controller used two input two output (TITO) system, and has demonstrated a better ability to track set-point changes when compared to IMC-tuned and Z-N tuned PID controllers. Shamiri et al. (2013) [11] utilized multiple input multiple output (MIMO) centralized model predictive control (MPC) to control polyethylene fluidized-bed reactor. Centralized MPC has proven to be able to control the reactor temperature and production rate successfully and effectively handled disturbance rejection and set point changes compared to PI controller.

As according to Ghasem (2006) [9], a hybrid controller (FLC-PI) was superior than advance controller (FLC) in controlling the polymerization reactors. Fuzzy control exhibited superior performance in transient state while PI has the advantage in controlling the steady state. The combination of two controllers was able to tackle the control problem effectively plus showing an excellent controller performance. Taking that into consideration, to control the reactor temperature, a Fuzzy-GMC hybrid controller is proposed in the current study to cope with the complexity of the non-linear process. A comparative study has been done with the conventional PID controller as well as GMC controller to compare their performance in both servo and regulatory control. An open loop analysis is also done to verify the model.

\section{Mathematical Model}

In this study, a modified model developed by Shamiri et al. (2015) [12] is chosen to represent the copolymerization process. It is a modified form of the two-phase model developed by Shamiri et al. (2011) [13]. Solid entrainment at the top of the reactor has been taken into account in this model. Generally, only small particles are regarded to be entrained and removed from the system while coarse particles will just stay within the system. But, there exists a situation whereby the velocities are much higher than the terminal velocity that could cause the coarse particles to be entrained from the reactor and the small particles stay. This phenomenon which is known as elutriation is also considered along with solid entrainment in the modified two-phase model.

The assumptions for this model are summarized as below:

a) The emulsion phase does not maintain at the minimum fluidization temperature, and it is assumed to be well-mixed. 
b) Polymerization reaction occur in both emulsion and bubble phase.

c) Constant bubble size is considered.

d) Heat and mass transfer resistances between solid and gas in both phases are negligible.

e) Uniform particle size throughout the bed.

f) Solid entrainment is considered at the top of the reactor

Based on the assumptions, the material balances for the system are as follows:

For emulsion phase:

$\left[M_{i}\right]_{e, i n} U_{e} A_{e}-\left[M_{i}\right]_{e} U_{e} A_{e}-R_{v}\left[M_{i}\right]_{e}+$

$\left(\left[M_{i}\right]_{b}-\left[M_{i}\right]_{e}\right) V_{e}\left(\frac{\delta}{1-\delta}\right)-\left(1-\varepsilon_{e}\right) R_{i, e}-$

$\frac{K_{e} V_{e} \varepsilon_{e} A_{e}\left[M_{i}\right]_{e}}{W_{e}}=\frac{d}{d t}\left(V_{e} \varepsilon_{e}\left[M_{i}\right]_{e}\right)$

For bubble phase:

$\left[M_{i}\right]_{b, i n} U_{b} A_{b}-\left[M_{i}\right]_{b} U_{b} A_{b}-R_{v} \varepsilon_{b}\left[M_{i}\right]_{b}-$ $K_{b e}\left(\left[M_{i}\right]_{b}-\left[M_{i}\right]_{e}\right) V_{b}$

$-\left(1-\varepsilon_{b}\right) \frac{A_{b}}{V_{P F R}} \int R_{i, b} d z-\frac{K_{b} V_{b} \varepsilon_{b} A_{b}\left[M_{i}\right]_{b}}{W_{b}}=$ $\frac{d}{d t}\left(V_{b} \varepsilon_{b}\left[M_{i}\right]_{b}\right)$

The energy balances are as below:

For emulsion phase:

$U_{e} A_{e}\left(T_{e, i n}-T_{\text {ref }}\right) \sum_{i=1}^{m}\left[M_{i}\right]_{e} C_{p, i}$

$U_{e} A_{e}\left(T_{e}-T_{r e f}\right) \sum_{i=1}^{m}\left[M_{i}\right]_{e} C_{p, i}-R_{v}\left(T_{e}-\right.$

$\left.T_{\text {ref }}\right)\left(\sum_{i=1}^{m} \varepsilon_{e}\left[M_{i}\right]_{e} C_{p, i}+(1-\right.$

$\left.\left.\varepsilon_{b}\right) \rho_{p o l} C_{p, p o l}\right)+\left(1-\varepsilon_{b}\right) R_{p, e} \Delta H_{R}-$

$H_{b e} V_{e}\left(\frac{\delta}{1-\delta}\right)\left(T_{e}-T_{b}\right)-V_{e} \varepsilon_{e}\left(T_{e}-\right.$

$\left.T_{\text {ref }}\right) \sum_{i=1}^{m} C_{p, i} \frac{d}{d t}\left(\left[M_{i}\right]_{e}\right)-$

$\frac{K_{e} A_{e}}{W_{e}\left(T_{e}-T_{r e f}\right)}\left(\sum_{i=1}^{m} \varepsilon_{e}\left[M_{i}\right]_{e} C_{p, i}+\right.$

$\left.\left(1-\varepsilon_{b}\right) \rho_{\text {pol }} C_{p, p o l}\right)=$

$V_{e}\left(\sum_{i=1}^{m} \varepsilon_{e}\left[M_{i}\right]_{e} C_{p, i}+\right.$

$\left.\left(1-\varepsilon_{b}\right) \rho_{p o l} C_{p, p o l}\right) \frac{d}{d t}\left(T_{e}-T_{r e f}\right)$

For bubble phase:

$U_{b} A_{b}\left(T_{b, i n}-T_{\text {ref }}\right) \sum_{i=1}^{m}\left[M_{i}\right]_{b} C_{p, i}-$

$U_{b} A_{b}\left(T_{b}-T_{\text {ref }}\right) \sum_{i=1}^{m}\left[M_{i}\right]_{b} C_{p, i}-R_{v}\left(T_{b}-\right.$

$\left.T_{\text {ref }}\right)\left(\sum_{i=1}^{m} \varepsilon_{b}\left[M_{i}\right]_{b} C_{p, i}+\right.$

$\left.\left(1-\varepsilon_{b}\right) \rho_{\text {pol }} C_{p, p o l}\right)+$

$\left(1-\varepsilon_{b}\right) \frac{A_{b} \Delta H_{R}}{V_{P F R}} \int R_{p, b} d z-H_{b e} V_{b}\left(T_{e}-T_{b}\right)-$

$V_{b} \varepsilon_{b}\left(T_{b}-T_{r e f}\right) \sum_{i=1}^{m} C_{p, i} \frac{d}{d t}\left(\left[M_{i}\right]_{b}\right)-$

$\frac{K_{b} A_{b}}{W_{b}\left(T_{b}-T_{r e f}\right)}\left(\sum_{i=1}^{m} \varepsilon_{b}\left[M_{i}\right]_{b} C_{p, i}+(1-\right.$

$\left.\left.\varepsilon_{b}\right) \rho_{\text {pol }} C_{p, p o l}\right)=V_{b}\left(\sum_{i=1}^{m} \varepsilon_{b}\left[M_{i}\right]_{b} C_{p, i}+\right.$

$\left.\left(1-\varepsilon_{b}\right) \rho_{p o l} C_{p, p o l}\right) \frac{d}{d t}\left(T_{b}-T_{r e f}\right)$
Where

$$
\begin{gathered}
K_{e}=23.7 \rho_{g} U_{o} \frac{A}{W_{e}} \exp \left(\frac{-5.4 U_{t} d_{p}}{U_{o}}\right) \\
K_{b}=23.7 \rho_{g} U_{o} \frac{A}{W_{b}} \exp \left(\frac{-5.4 U_{t} d_{p}}{U_{o}}\right) \\
W_{e}=A H\left(1-\varepsilon_{e}\right) \rho_{p o l} \\
W_{b}=A H\left(1-\varepsilon_{b}\right) \rho_{p o l} \\
U_{t}=U_{t}^{*}\left[\mu \rho_{g}{ }^{-2}\left(\rho_{p o l}-\rho_{g}\right) g\right]^{1 / 3} \\
U_{t}^{*}= \\
\text { for } 0.5<\emptyset_{s} \leq 1 \\
d_{p}^{*}=d_{p}\left[\mu^{-2} \rho_{g}\left(\rho_{p o l}-\rho_{g}\right) g\right]^{1 / 3}
\end{gathered}
$$

Equations (1) to (4) are solved by using MATLAB with the following initial conditions:

$$
\begin{gathered}
{\left[M_{i}\right]_{b, t=0}=\left[M_{i}\right]_{i n}} \\
T_{b}(t=0)=T_{i n} \\
{\left[M_{i}\right]_{e, t=0}=\left[M_{i}\right]_{i n}} \\
T_{e}(t=0)=T_{i n}
\end{gathered}
$$

\begin{tabular}{|c|c|c|}
\hline Parameters & Formula & Reference \\
\hline $\begin{array}{l}\text { Minimum } \\
\text { fluidization } \\
\text { velocity }\end{array}$ & $\begin{array}{l}R e_{m f} \\
=\left[(29.5)^{2}+0.375 A r\right]^{1 / 2} \\
-29.5\end{array}$ & [14] \\
\hline $\begin{array}{l}\text { Bubble } \\
\text { velocity }\end{array}$ & $U_{b}=U_{o}-U_{m f}-U_{b r}$ & \\
\hline $\begin{array}{l}\text { Bubble rise } \\
\text { velocity }\end{array}$ & $U_{b r}=0.711\left(g d_{b}\right)^{1 / 2}$ & [15] \\
\hline $\begin{array}{l}\text { Emulsion } \\
\text { velocity }\end{array}$ & $U_{e}=\frac{U_{m f}}{\varepsilon_{m f}(1-\delta)}$ & \\
\hline $\begin{array}{l}\text { Bubble } \\
\text { diameter }\end{array}$ & $\begin{array}{l}\text { For Geldart B: } \\
d_{b}=d_{b 0}\left[1+27\left(U_{0}-U_{e}\right)\right]^{\frac{1}{3}}(1+ \\
6.84 H) d_{b 0}=0.0085\end{array}$ & [16] \\
\hline $\begin{array}{l}\text { Mass } \\
\text { transfer } \\
\text { coefficient }\end{array}$ & $\begin{array}{l}K_{b e}=\left(\frac{1}{K_{b c}}+\frac{1}{K_{c e}}\right)^{-1} \\
K_{b c}=4.5\left(\frac{U_{e}}{d_{b}}\right)+ \\
5.85\left(\frac{D_{g}^{1 / 2} g^{1 / 4}}{d_{b}^{5 / 4}}\right) \\
K_{c e}=6.77\left(\frac{D_{g} \varepsilon_{e} U_{b r}}{d_{b}}\right)\end{array}$ & [15] \\
\hline
\end{tabular}

All correlations and equations related to this modified two-phase model are listed in Table 1 below.

Table 1. Correlations and equations used in the mathematical model 


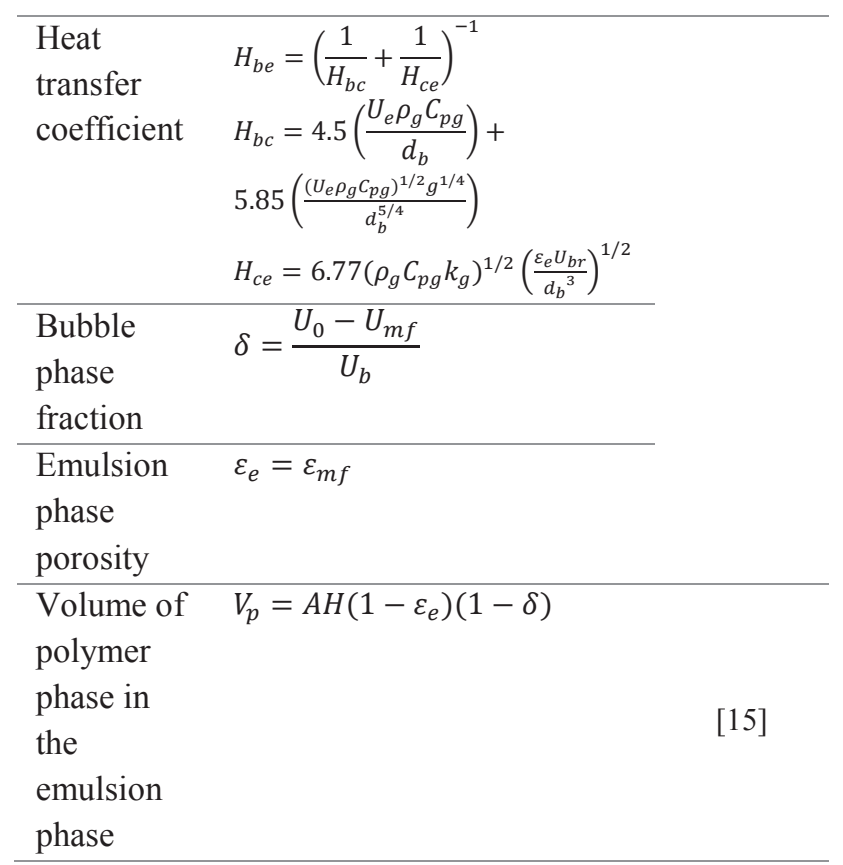

\section{Control Strategy}

As mentioned in the previous studies on the temperature control of the polymerization process, in order to tackle this non-linearity issue, a hybrid controller is suggested to be better than other types of controller when it comes to coping with the complex system. In this work, a fuzzy-GMC hybrid controller is designed to control the reactor temperature by manipulating the cooling water valve rate, $\mathrm{F}_{\mathrm{c}}(\%)$.

\subsection{Generic Model Control}

GMC is very highly dependent on the model and is only limited for the systems with linear in control.

GMC control algorithms are given by:

$K_{1} \varepsilon(t)+\int_{0}^{t_{f}} K_{2} \varepsilon(t) d t=\frac{\partial H(X)}{\partial x} \times F(X, U, t)$

Where $\mathrm{H}(\mathrm{x})$ is the controlled variable.

For any non-linear system with linear control,

$$
F(X, U, t)=F(X)+G(X) U
$$

Therefore,

$K_{1} \varepsilon(t)+\int_{0}^{t_{f}} K_{2} \varepsilon(t) d t=\frac{\partial H(X)}{\partial x} \times[F(X)+G(X) U]$

In this case, $\frac{\partial H(X)}{\partial x}=1$.

Hence, the GMC formulation is given by,

$$
U=\frac{K_{1} \varepsilon(t)+\int_{0}^{t_{f}} K_{2} \varepsilon(t) d t-F(X)}{G(X)}
$$

In implementing the above strategy for the propylene copolymerization process, $F(X)$ and $G(X)$ are mass and energy balances of the emulsion phase in Equation (3), $U$ is the coolant flow $\left(F_{c}\right)$, which is the manipulated variable to control the reactor temperature, and $\varepsilon(t)$ is the difference between the emulsion temperature and the desired set point. Hence, for the current process, GMC formulation becomes as follows:

$$
F_{c}=\frac{\propto\left[K_{1} \varepsilon(t)+\int_{0}^{t_{f}} K_{2} \varepsilon(t) d t\right]-F(X)}{G(X)}
$$

where

$$
\begin{aligned}
& \propto=V_{e}\left(\sum_{i=1}^{m} \varepsilon_{e}\left[M_{i}\right]_{e} C_{p, i}+\left(1-\varepsilon_{b}\right) \rho_{p o l} C_{p, p o l}\right) \\
& \left(1-\varepsilon_{b}\right) R_{p, e} \Delta H_{R}+H_{b e} V_{e}\left(\frac{\delta}{1-\delta}\right)\left(T_{e}-T_{b}\right) \\
& +U_{e} A_{e}\left(T_{e}-T_{r e f}\right) \sum_{i=1}^{m}\left[M_{i}\right]_{e} C_{p, i} \\
& +R_{v}\left(T_{e}-T_{r e f}\right)\left(\sum_{i=1}^{m} \varepsilon_{e}\left[M_{i}\right]_{e} C_{p, i}+\left(1-\varepsilon_{b}\right) \rho_{p o l} C_{p, p o l}\right) \\
& F(X)= \\
& +V_{e} \varepsilon_{e}\left(T_{e}-T_{r e f}\right) \sum_{i=1}^{m} C_{p, i} \frac{d}{d t}\left(\left[M_{i}\right]_{e}\right) \\
& +\frac{K_{e} A_{e}}{W_{e}\left(T_{e}-T_{r e f}\right)}\left(\sum_{i=1}^{m} \varepsilon_{e}\left[M_{i}\right]_{e} C_{p, i}+\left(1-\varepsilon_{b}\right) \rho_{p o l} C_{p, p o l}\right) \\
& +\left(333.15-T_{r e f}\right)\left(U_{e} A_{e} \sum_{i=1}^{m}\left[M_{i}\right]_{e} C_{p, i}\right) \\
& G(X)=\frac{K e^{-\tau_{D} S}}{\tau s+1} U_{e} A_{e} \sum_{i=1}^{m}\left[M_{i}\right]_{e} C_{p, i}
\end{aligned}
$$

GMC performance specifications, $\mathrm{K}_{1}$ and $\mathrm{K}_{2}$ is obtained from the generalized GMC profile specification graph by Lee and Sullivan (1988) [17]. These values are selected based on the desired response suitable for the process. For the copolymerization process, $\mathrm{K}_{1}=9 \times 10^{-3}$ and $\mathrm{K}_{2}=$ $2.25 \times 10^{-6}$.

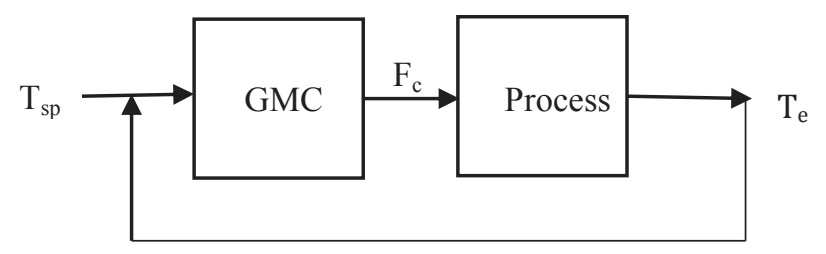

FIGURE 2. GMC control scheme

\subsection{Fuzzy-GMC Hybrid Control}

The purpose for the hybrid control scheme is to modify the outcome from GMC, in order to reduce the overshoot problem. A fuzzy-logic controller is used to do the modification then coupled with GMC to control the reactor.

FLC is strongly based on its fuzzy sets, linguistic variables and the approximate reasoning. A set of fuzzy rules are set based on the input and output variables. Its input variables are error, $e$ (the difference between setpoint and reactor temperature) and $\Delta T_{e} / F_{c}$ (quotient of the changes of the reactor temperature and the associated coolant valve rate). The output variable is a factor, $\mathrm{f}$ where this value is used to compensate the outcome from $\operatorname{GMC}\left(\Delta \mathrm{F}_{\mathrm{c}}=\mathrm{f} \times \mathrm{F}_{\mathrm{c}}\right)$. 
This is a simple designed fuzzy logic controller by using Mamdani method. The membership functions are in triangular form.

TABLE 2. Linguistic terms for process variables

\begin{tabular}{|c|c|c|}
\hline \multicolumn{2}{|c|}{ Process variables } & Linguistic terms \\
\hline \multirow{6}{*}{ Input } & \multirow{3}{*}{$e$} & Positive (P) \\
\hline & & Negative (N) \\
\hline & & Zero $(Z)$ \\
\hline & \multirow{3}{*}{$\Delta T_{e} / F_{c}$} & Small (Sm) \\
\hline & & Medium (Med) \\
\hline & & Large (L) \\
\hline \multirow{7}{*}{ Output } & \multirow{7}{*}{$f$} & Negative large \\
\hline & & Negative medium \\
\hline & & Negative small \\
\hline & & Positive large \\
\hline & & Positive medium \\
\hline & & Positive small \\
\hline & & Zero \\
\hline
\end{tabular}

The fuzzy rules are:

1. IF (error is negative) AND $\left(\Delta \mathrm{T}_{\mathrm{e}} / \mathrm{F}_{\mathrm{c}}\right.$ is small) THEN (f is negative large)

2. IF (error is negative) AND $\left(\Delta \mathrm{T}_{\mathrm{e}} / \mathrm{F}_{\mathrm{c}}\right.$ is medium) THEN ( $\mathrm{f}$ is negative medium)

3. IF (error is negative) AND $\left(\Delta \mathrm{T}_{\mathrm{e}} / \mathrm{F}_{\mathrm{c}}\right.$ is large) THEN ( $\mathrm{f}$ is negative small)

4. IF (error is positive) AND $\left(\Delta \mathrm{T}_{\mathrm{e}} / \mathrm{F}_{\mathrm{c}}\right.$ is small) THEN ( $\mathrm{f}$ is positive large)

5. IF (error is positive) AND $\left(\Delta \mathrm{T}_{\mathrm{e}} / \mathrm{F}_{\mathrm{c}}\right.$ is medium) THEN ( $\mathrm{f}$ is positive medium)

6. IF (error is positive) AND $\left(\Delta \mathrm{T}_{\mathrm{e}} / \mathrm{F}_{\mathrm{c}}\right.$ is large) THEN ( $\mathrm{f}$ is positive small)

7. IF (error is zero) THEN ( $\mathrm{f}$ is zero)

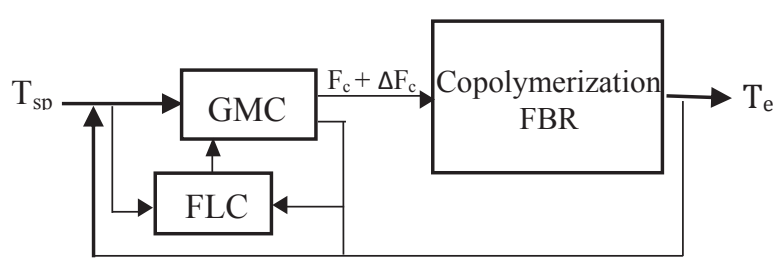

Fig 3. Fuzzy-GMC hybrid control scheme

\section{RESULTS AND DISCUSSION}

\subsection{Open-Loop Analysis}

To verify the constructed mathematical model, the process is simulated in MATLAB. Several operating conditions (superficial velocity, propylene and ethylene feed concentration, coolant valve rate) were subjected to step changes and Figure 4 to 7 shows the effect of step change in each parameter to the emulsion temperature.

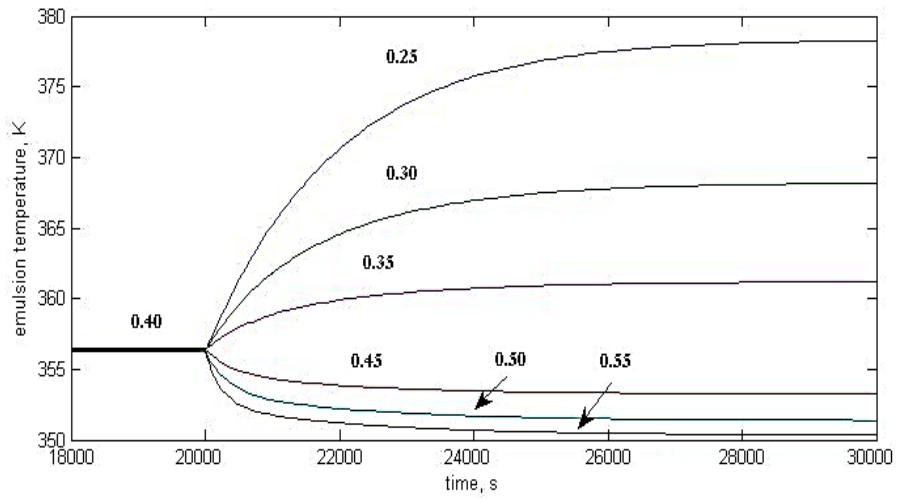

Fig 4. Effect of step change in superficial velocity, U0 on the emulsion temperature.

Based on Figure 4, the step change in superficial velocity shows a significant impact on the emulsion temperature. The nominal value of superficial velocity is taken as $0.4 \mathrm{~m} / \mathrm{s}$. In order to demonstrate the nonlinearity, step changes are taken $0.05 \mathrm{~m} / \mathrm{s}$ larger or smaller than the nominal value. Emulsion temperature increases with decreasing the superficial velocity. As shown in the figure, even with a very small change in the superficial velocity, the impact on the emulsion temperature was large and this indicates that it is crucial to monitor the superficial velocity when we are controlling the temperature of the reactor. Since the figure above shows a non-symmetry response, this shows that the relationship is non-linear.

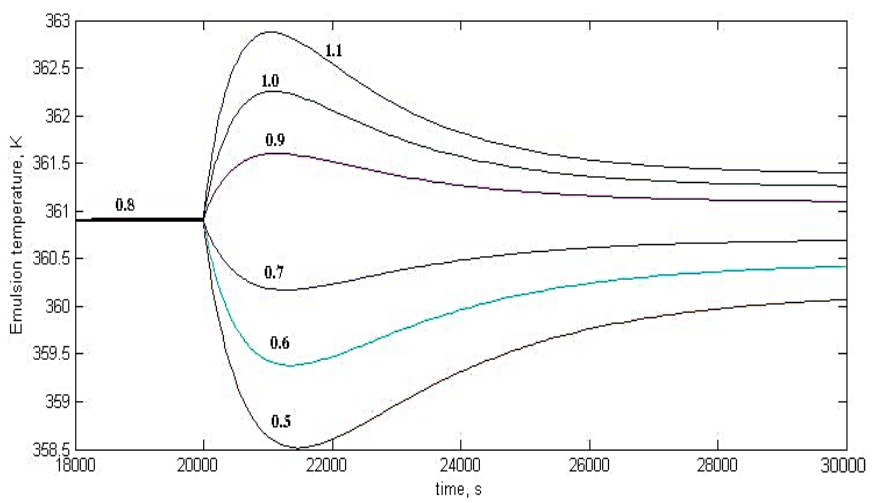

Fig 5. Effect of step change in the propylene concentration on the emulsion temperature.

Effect of monomer concentration on the emulsion temperature is depicted in Figure 5. It can be seen in this figure that changes in the propylene concentration has a significant impact on the reactor temperature when the concentration is changed by $0.1 \mathrm{~mol} / \mathrm{L}$ from the nominal value. The non-symmetrical pattern in this figure indicates that the reactor temperature changes nonlinearly with change of propylene concentration. Hence, it can be concluded that the propylene concentration is among the important parameters when controlling the reactor temperature. 


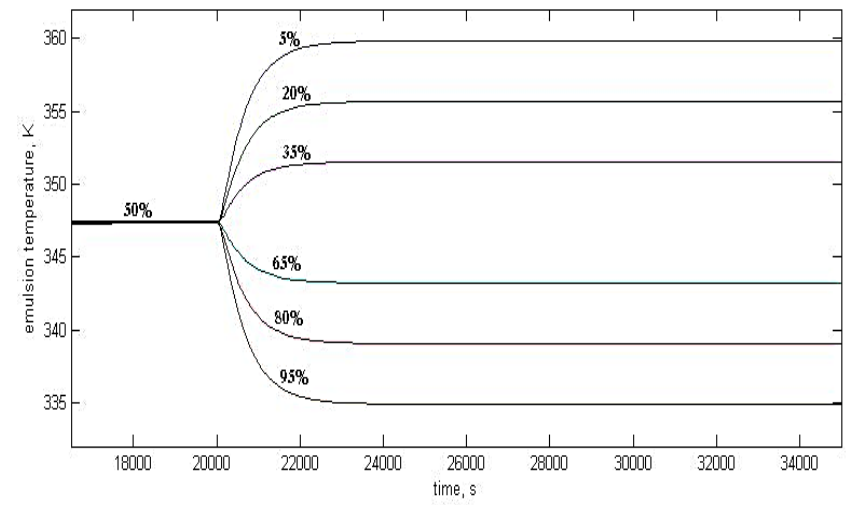

Fig 6. Effect of step change in coolant flow (\%) on emulsion temperature

Figure 6 illustrate the effect of step change in coolant valve rate $(\%)$ on the emulsion temperature. Change in valve rate should have linear relationship with emulsion temperature in order to directly change the temperature when needed. As shown in figure, the symmetrical pattern shows the linear relationship.

Based on these analysis, it can be said that the model is reliable, and a dependable representative of the copolymerization process.

\subsection{Control System Analysis}

Conventional controller, PID is also designed to compare its performance. By using Ziegler-Nichols method, the PID setting are as follows:

$\mathrm{K}_{\mathrm{c}}=24.24$

$\tau_{\mathrm{I}}=129.5$

$\tau_{\mathrm{D}}=32.37$

\subsubsection{Set-Point Tracking}

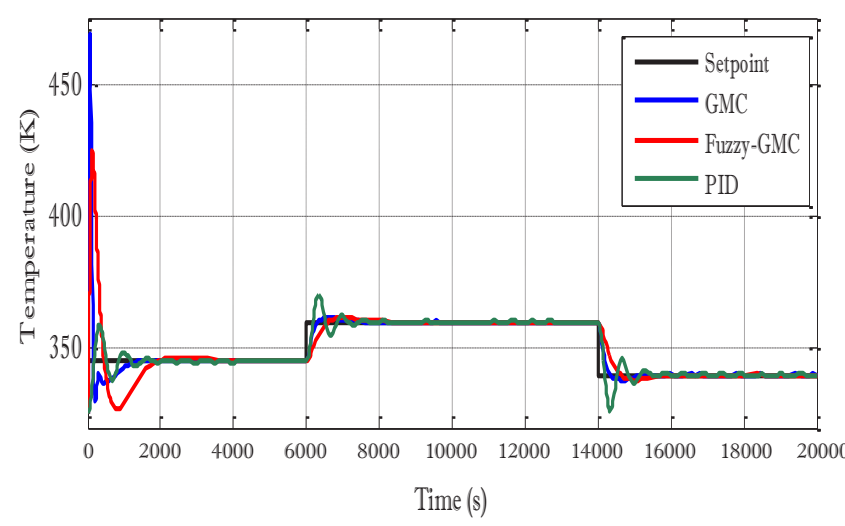

Fig 7. Performance comparison of GMC, Fuzzy-GMC Hybrid and PID controller in set-point tracking.

Figure 7 shows the performances of the controllers tracking the set-point changes. At the first part, GMC shows a very high overshoot and a very good set-point tracking. However, fuzzy-GMC controller manages to track the change in set-point in less than 1000 secs and also reduces the overshoot. PID on the other hand, gives low overshoot but exhibit poor performance in tracking the set-point changes compared to the other two controllers. Hence, hybrid controller exhibit better performance than GMC and PID controllers (low overshoot and very fast response).

\subsubsection{Disturbance Rejection}

Two disturbances are introduced to the system to analyze the disturbance rejection capability for each controller. Changes in superficial velocity, $\mathrm{U}_{0}$ from its nominal value $(0.35 \mathrm{~m} / \mathrm{s})$ are imposed to the system. At 4000 secs, $\mathrm{U}_{0}$ is increased to $0.55 \mathrm{~m} / \mathrm{s}$ and then decreased to $0.3 \mathrm{~m} / \mathrm{s}$ at $8000 \mathrm{secs}$. Changes in ethylene concentration is also applied to the process. At 6000 secs, the concentration is increased from its nominal value of 0.05 $\mathrm{mol} / \mathrm{L}$ to $0.15 \mathrm{~mol} / \mathrm{L}$ and then decreased to $0.03 \mathrm{~mol} / \mathrm{L}$ at 14000 secs.

Based on Figure 8, PID controller is unable to cope with the disturbance since the response did not stop oscillating. Although the overshoot is low, the oscillating response did not exhibit the capability of the PID to reject the disturbance well. Both GMC and fuzzy-GMC hybrid controller exhibit good performance in rejecting the disturbances. The slight difference is observed that is fuzzy-GMC hybrid controller's response is much stable after the disturbance is introduced compared to GMC controller.

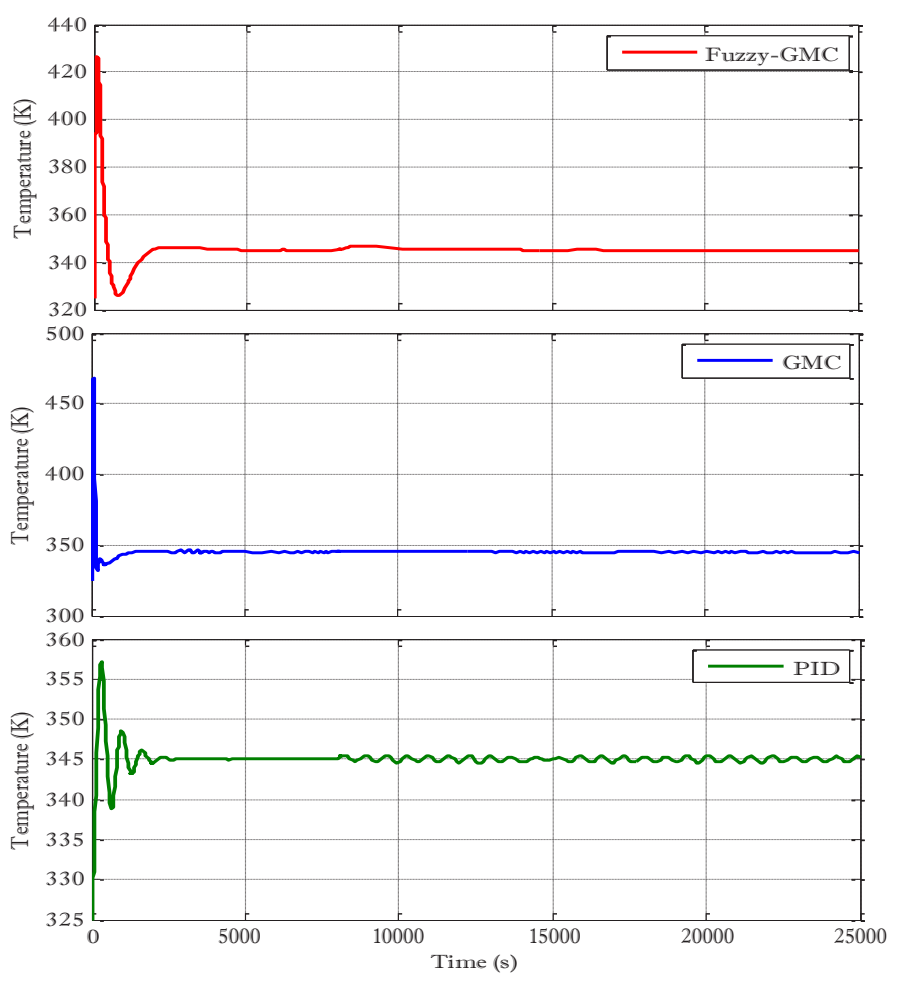

Fig 8. Controllers' performance in disturbance rejection.

\section{Conclusion}

A modified two-phase model that considered solid entrainment in the fluidized bed reactor is utilized in the study to represent the copolymerization process. The model is then verified by using open-loop analysis. The 
analysis showed that the model is reliable and a dependable representation of the process.

A model based controller, GMC is designed to control the temperature of the reactor. GMC gave a very high overshoot in its response. A hybrid controller is then designed to reduce the overshoot. Results show that fuzzy-GMC hybrid controller is able to reduce the overshoot problem and provide the best response in set-

\section{References}

1. Richards, J. R., \& Congalidis, J. P., Comput. Chem. Eng., 30(10-12), 1447-1463 (2006).

2. Ali, M. A.-H., Betlem, B., Weickert, G., \& Roffel, B., Chem. Eng. Process, 46(6), 554-564 (2007).

3. Ho, Y. K., Shamiri, A., Mjalli, F. S., \& Hussain, M. A., J. Process Contr., 22(6), 947-958 (2012).

4. Ibrehem, A. S., Hussain, M. A., \& Ghasem, N. M. Chinese J. Chem. Eng., 16(1), 84-89 (2008).

5. Salau, N. P. G., Neumann, G. A., Trierweiler, J. O., \& Secchi, A. R., Ind. Eng. Chem. Res., 47(16), 6058-6069 (2008).

6. Vahidi, O., Shahrokhi, M., \& Mirzaei, A., Iran. Chem. Chem. Eng., 27(3), 87-101 (2008).

7. Dadebo, S. A., Bell, M. L., McLellan, P. J., \& McAuley, K. B., J. Process Contr., 7(2), 83-95 (1997).

8. Ali, E., Al-Humaizi, K., \& Ajbar, A., Ind. Eng. Chem. Res., 42(11), 2349-2364 (2003).

9. Ghasem, N. M., Chem. Eng. Res. Des., 84(2), 97106 (2006)

10. Ali, E. M., \& Ali, M. A.-H., ISA T, 49(1), 177187 (2010).

11. Shamiri, A., Hussain, M. a., Mjalli, F. s., Mostoufi, N., \& Hajimolana, S., Chinese J. Chem. Eng., 21(9), 1015-1029 (2013).

12. Shamiri, A., Wong, S. W., Zanil, M. F., Hussain, M. A., \& Mostoufi, N., Chem. Eng. J., 264, 706719 (2015).

13. Shamiri, A., Azlan Hussain, M., Sabri Mjalli, F., Mostoufi, N., \& Saleh Shafeeyan, M., Chem. Eng. Sci., 66(6), 1189-1199 (2011).

14. Lucas, A., Arnaldos, J., Casal, J., \& Puigjaner, L., Ind. Eng. Chem. Process Des. Dev., 25(2), 426429 (1986).

15. Kunii, D., \& Levenspiel, O., Fluidization Engineering: Butterworth-Heinemann (1991). point tracking as well as disturbance rejection compared to GMC and PID controller. Future study will be needed to further tune the hybrid controller to improve the initial overshoot problem.

The authors acknowledge the funding from FRGS (FP0642015A) University of Malaya.

16. Hilligardt, K., \& Werther, J., Chem-Ing-Tech, 57(7), 622-623 (1985).

17. Lee, P. L., \& Sullivan, G. R. (1988). Generic model control (GMC). Computers \& Chemical Engineering, 12(6), 573-580.

18. Alizadeh, M., Mostoufi, N., Pourmahdian, S., \& Sotudeh-Gharebagh, R., Chem. Eng. J, 97(1), $27-$ 35 (2004).

19. Chatzidoukas, C., Perkins, J. D., Pistikopoulos, E. N., \& Kiparissides, C., Chem. Eng. Sci., 58(16), 3643-3658 (2003).

20. Choi, K. Y., \& Ray, W. H., Chem. Eng. Sci., 40(12), 2261-2279 (1985)

21. Cui, H., Mostoufi, N., \& Chaouki, J., Chem. Eng. J., 79(2), 133-143 (2000).

22. Cui, H., Mostoufi, N., \& Chaouki, J., Powder Technol, 120(1-2), 12-20 (2001).

23. Hatzantonis, H., Yiannoulakis, H., Yiagopoulos, A., \& Kiparissides, C., Chem. Eng. Sci., 55(16), 3237-3259 (2000).

24. Ibrehem, A. S., Hussain, M. A., \& Ghasem, N. M., Aust. J. Basic \& Appl., 3(4), 3954-3974 (2009).

25. Ibrehem, A. S., Hussain, M. A., \& Ghasem, N. M., Chem. Eng. J., 149(1-3), 353-362 (2009).

26. Ibrehem, A. S., Hussain, M. A., \& Ghasem, N. M., Iran. Chem. Chem. Eng., 31(4), 91-117 (2012).

27. McAuley, K. B., \& Macgregor, J. F., Aiche J., 39(5), 855-866 (1993).

28. McAuley, K. B., Talbot, J. P., \& Harris, T. J., Chem. Eng. Sci., 49(13), 2035-2045 (1994).

29. Shamiri, A., Hussain, M. A., Mjalli, F. S., \& Mostoufi, N., Chem. Eng. J., 161(1-2), 240-249 (2010).

30. Shamiri, A., Hussain, M. A., Mjalli, F. S., \& Mostoufi, N., Comput. Chem. Eng., 36(0), 35-47 (2012). 